A. B. Kharazishvili, A. Razmadze Institute of Mathematics, M. Alexidze Street, 1, Tbilisi 0193, Republic of Georgia. email: kharaz2@yahoo.com

A. P. Kirtadze, I. Vekua Institute of Applied Mathematics, University Street, 2, Tbilisi 0143, Republic of Georgia. email: kirtadze2@yahoo.com

\title{
ON WEAKLY METRICALLY TRANSITIVE MEASURES AND NONMEASURABLE SETS
}

\begin{abstract}
It is shown that some analog of Minkowski's method in geometric number theory enables to establish the existence of sets which are absolutely nonmeasurable with respect to weakly metrically transitive invariant measures.
\end{abstract}

In 1896, Minkowski published his famous work [7] in which he presented a geometric approach to classical problems of number theory and extensively developed beautiful geometric methods in this theory. The main role in his methods was played by convex subsets of Euclidean spaces. Among many other important results, Minkowski proved in [7] the following fundamental theorem: if $C$ is a bounded convex body in the Euclidean space $\mathbb{R}^{n}$, symmetric with respect to the origin of $\mathbb{R}^{n}$ and having volume greater than or equal to $2^{n}$, then $C$ contains at least two nonzero points of the lattice $\mathbb{Z}^{n}$, where $\mathbb{Z}$ denotes the set of all integers. This statement was then generalized in various directions (see, e.g., [3] and references therein).

The argument used by Minkowski in the proof of his theorem is purely group-theoretic and measure-theoretic. In fact, by applying a similar argument, the existence of a subset of $\mathbb{R}^{n}$ nonmeasurable in the Lebesgue sense can be established. In this context, it is interesting to note that the rigorous concept of the Lebesgue measure was introduced some years later after Minkowski's theorem was stated, namely, in the beginning of the twentieth

Key Words: Invariant measure, nonmeasurable set, convex set, weakly metrically transitive measure, extension of measure, $G$-selector.

Mathematical Reviews subject classification: Primary: 28A05; Secondary: 28D05.

Received by the editors September 20, 2006

Communicated by: Krzysztof Chris Ciesielski 
century. Moreover, recall that the existence of subsets of $\mathbb{R}^{n}$, which are nonmeasurable in the Lebesgue sense, was first proved by Vitali [11] only in 1905.

An analogous situation can be observed in connection with the famous Poincaré theorem on recurrent points for a finite measure space $(E, \mu)$ equipped with its transformation $g: E \rightarrow E$ which preserves the given measure $\mu$. The above-mentioned theorem was proved by Poincaré before the basic concepts of measure theory (e.g., the countable additivity of measures) were introduced in real analysis (cf. [8], Chapter 17).

The main goal of this paper is to demonstrate the role of Minkowski's method in establishing the existence of nonmeasurable subsets of Euclidean spaces and, more generally, in establishing the existence of nonmeasurable sets in an abstract space equipped with a transformation group and some measure, which is invariant under that group. In the sequel, we will introduce the concept of a weakly metrically transitive invariant measure and will show how such a measure produces absolutely nonmeasurable sets.

We begin with several auxiliary notions concerning spaces endowed with transformation groups.

Let $E$ be a nonempty set, $G$ be a group of transformations of $E$, and let $\mu$ be a complete measure defined on some $\sigma$-algebra of subsets of $E$ and invariant under all transformations from $G$ (we shall say, in short, that $\mu$ is a $G$-invariant measure). Let $X$ be a subset of $E$.

We shall say that $X$ is $G$-thick with respect to $\mu$ if there exists a countable subgroup $H$ of $G$ such that

$$
\mu(E \backslash \cup\{h(X): h \in H\})=0 .
$$

We shall say that $X$ is $G$-thin with respect to $\mu$ if

$$
\mu(g(X) \cap h(X))=0 \quad(g \in G, h \in G, g \neq h) .
$$

In particular, if a group $G$ acts freely in a space $E$, then every singleton is $G$-thin in $E$.

Example 1. Suppose that $\mu$ is a $\sigma$-finite $G$-invariant measure on $E$ metrically transitive with respect to $G$; i.e., for any $\mu$-measurable set $Z$, the relation

$$
(\forall g \in G)(\mu(g(Z) \triangle Z)=0)
$$

implies $\mu(Z)=0$ or $\mu(E \backslash Z)=0$. Then it can easily be checked that every $\mu$ measurable set $X$ with $\mu(X)>0$ is $G$-thick in $E$. In this context, let us recall that the left Haar measure on a $\sigma$-compact locally compact topological group $(H, \cdot)$ is metrically transitive with respect to the group of all left translations of $H$. 
Example 2. Suppose that a group $G$ of transformations of a given space $E$ is countable and suppose, in addition, that $G$ acts almost freely in $E$ with respect to a given $G$-invariant measure $\mu$; i.e., for any two distinct transformations $g \in G$ and $h \in G$, we have

$$
\mu(\{x \in E: g(x)=h(x)\})=0 .
$$

Consider the partition of $E$ into all $G$-orbits of points of $E$. Let $X$ be an arbitrary selector of this partition (in short, $G$-selector). Then it is not difficult to verify that $X$ is simultaneously $G$-thick and $G$-thin in $E$.

The following statement is essentially due to Minkowski (cf. [7]).

Theorem 1. Let $\mu$ be a $\sigma$-finite $G$-invariant measure on $E$. Let $X$ be a $G$ thick subset of $E$, and let $Y$ be a $G$-thin subset of $E$. If both of these sets are measurable with respect to $\mu$, then $\mu(Y) \leq \mu(X)$.

Proof. If the group $G$ is uncountable, then there is nothing to prove because the $\mu$-measurable $G$-thin subset $Y$ of $E$ is necessarily of $\mu$-measure zero (in view of the $\sigma$-finiteness and $G$-invariance of $\mu$ and the almost disjointedness of the family $\{g(Y): g \in G\})$.

It remains to consider the case when $G$ is countable. In this case, we obviously have $\mu(E \backslash \cup\{g(X): g \in G\})=0$, and, consequently,

$$
\begin{aligned}
\mu(Y) & =\mu(Y \cap(\cup\{g(X): g \in G\})) \leq \sum_{g \in G} \mu(g(X) \cap Y) \\
& =\sum_{g \in G} \mu(X \cap g(Y)) \leq \mu(X)
\end{aligned}
$$

which yields the required result.

Let $\mu$ be a $\sigma$-finite $G$-invariant measure on $E$. We shall say that $\mu$ is weakly metrically transitive with respect to $G$ if, for any $\varepsilon>0$, there exist a $\mu$-measurable set $X$ with $\mu(X)<\varepsilon$ and a countable subgroup $H$ of $G$ such that

$$
\mu(E \backslash \cup\{h(X): h \in H\})=0 .
$$

It is not hard to verify that any $\sigma$-finite nonatomic $G$-invariant metrically transitive measure $\mu$ on $E$ is weakly metrically transitive. The converse assertion is not true in general. Indeed, it directly follows from the above definition that every $G$-invariant extension of a $G$-invariant weakly metrically transitive measure is also weakly metrically transitive. An analogous statement fails to be true for $G$-invariant metrically transitive measures. 
Example 3. Let us take $E=\mathbb{R}, G=$ the group of all translations of $\mathbb{R}$, and $\mu=$ the Lebesgue measure on $\mathbb{R}$. Obviously, we may identify $G$ with the additive group $\mathbb{R}$. It is well known that $\mu$ is metrically transitive with respect to the group $\mathbb{Q} \subset \mathbb{R}$ of all rationals. By applying the standard transfinite methods (see, e.g., [8], [4]), it can be proved that there exist two subsets $A$ and $B$ of $\mathbb{R}$ satisfying the following conditions:

(1) $A \cap B=\emptyset, \quad A \cup B=\mathbb{R}$ and $\operatorname{card}(A)=\operatorname{card}(B)=\operatorname{card}(\mathbb{R})$;

(2) $\mu^{*}(A)=\mu^{*}(B)=+\infty$;

(3) both sets $A$ and $B$ are almost $\mathbb{R}$-invariant; i.e., for all $h \in \mathbb{R}$, we have $\operatorname{card}((h+A) \triangle A)<\operatorname{card}(\mathbb{R})$ and $\operatorname{card}((h+B) \triangle B)<\operatorname{card}(\mathbb{R})$.

By using these two sets, the original measure $\mu$ can be extended to an $\mathbb{R}$ invariant measure $\mu^{\prime}$ which is not metrically transitive with respect to the group $\mathbb{R}$ (hence, with respect to the group $\mathbb{Q} \subset \mathbb{R}$ ). For this purpose, it suffices to define on the $\sigma$-algebra generated by $\{A, B\} \cup \operatorname{dom}(\mu)$ the functional $\mu^{\prime}$ by the formula

$$
\mu^{\prime}((A \cap X) \cup(B \cap Y))=(1 / 2)(\mu(X)+\mu(Y)),
$$

where $X$ and $Y$ are arbitrary elements from $\operatorname{dom}(\mu)$. This definition of $\mu^{\prime}$ is correct, and $\mu^{\prime}$ is not metrically transitive in view of the almost invariance of the $\mu^{\prime}$-measurable sets $A$ and $B$, which both are of strictly positive $\mu^{\prime}$-measure.

Example 4. Suppose that a $\sigma$-finite $G$-invariant metrically transitive measure $\mu$ on $E$ has at least one atom. Then it is not difficult to describe the structure of $\mu$. Namely, in this case, the space $E$ admits a representation $E=\cup\left\{A_{i}: i \in I\right\}$ where

(1) the set $I$ is at most countable, and the family $\left\{A_{i}: i \in I\right\}$ is disjoint;

(2) each set $A_{i}(i \in I)$ is an atom of $\mu$;

(3) for any $i \in I$ and $j \in J$, there exists a transformation $g \in G$ such that $\mu\left(g\left(A_{i}\right) \triangle A_{j}\right)=0$

(4) for any transformation $h \in G$, the family $\left\{h\left(A_{i}\right): i \in I\right\}$ almost coincides with the family $\left\{A_{i}: i \in I\right\}$; i.e., each member of the first family almost coincides with some member of the second family, and conversely.

In other words, we obtain a certain $G$-invariant lattice of atoms of our measure $\mu$, similar to the standard lattice $\left\{g+[0,1]^{n}: g \in \mathbb{Z}^{n}\right\}$ of the Euclidean space $\mathbb{R}^{n}$.

Let $(E, G)$ be a space with a transformation group, and let $M$ be some class of $G$-invariant measures on $E$ (in general, we do not assume that measures from $M$ are defined on the same $\sigma$-algebra).

We shall say that a set $X \subset E$ is absolutely nonmeasurable with respect to $M$ if there exists no measure from $M$ for which $X$ is measurable. 
Let $\mu$ be a $G$-invariant measure on $E$. We shall say that a set $X \subset E$ is absolutely nonmeasurable with respect to $\mu$ if $X$ is absolutely nonmeasurable with respect to the class of all $G$-invariant measures on $E$ extending $\mu$.

The next example underlines a close relationship between convex sets and absolutely nonmeasurable sets.

Example 5. Let $E$ be an infinite-dimensional separable Banach space, $G$ be the group of all translations of $E$, and let $B$ be a bounded convex body in $E$. It can be proved that $B$ is absolutely nonmeasurable with respect to all nonzero $\sigma$-finite $G$-invariant (more generally, $G$-quasi-invariant) measures on $E$ (see [5]). This fact implies, in particular, that $E$ admits no nonzero $\sigma$-finite $G$-quasi-invariant Borel measure. By using an algebraic isomorphism between the additive groups $E$ and $\mathbb{R}$, it can also be shown that there exists a subset of $\mathbb{R}$ which is absolutely nonmeasurable with respect to the class of all nonzero $\sigma$-finite $\mathbb{R}$-invariant (more generally, $\mathbb{R}$-quasi-invariant) measures on $\mathbb{R}$.

Example 6. Let $E=\mathbb{R}, G=$ the group of all translations of $\mathbb{R}$, and $\mu=$ the Lebesgue measure on $\mathbb{R}$. Any selector of $\mathbb{R} / \mathbb{Q}$ is called a Vitali subset of $\mathbb{R}$. It is well known that all Vitali sets in $\mathbb{R}$ are absolutely nonmeasurable with respect to $\mu$ (see, e.g., [11], [8], [4]).

Theorem 2. Let $G$ be a countable group of transformations of $E$, and let $\mu$ be a nonzero $\sigma$-finite $G$-invariant weakly metrically transitive measure on $E$. Suppose also that $G$ acts almost freely in $E$ with respect to $\mu$. Then every $G$-selector is absolutely nonmeasurable with respect to $\mu$.

Proof. Take an arbitrary $G$-selector $X$ in $E$. We must check that, for any $G$-invariant measure $\mu^{\prime}$ on $E$ extending the original measure $\mu$, the set $X$ is $\mu^{\prime}$-nonmeasurable. Suppose otherwise; i.e., $X \in \operatorname{dom}\left(\mu^{\prime}\right)$. Then, in view of the countability of $G$ and the equality $E=\cup\{g(X): g \in G\}$, we have $\mu^{\prime}(X)>0$. Let us denote $\mu^{\prime}(X)=\varepsilon$. According to our assumption, there exist a $\mu$-measurable set $Y$ with $\mu(Y)<\varepsilon$ and a countable family $H \subset G$ such that $\mu(E \backslash \cup\{h(Y): h \in H\})=0$. In other words, $Y$ turns out to be a $G$-thick subset of $E$ with respect to $\mu$. Since $\mu^{\prime}$ extends $\mu$, the set $Y$ is $G$-thick with respect to $\mu^{\prime}$ as well. At the same time, the selector $X$ is a $G$-thin subset of $E$ with respect to $\mu$ and, hence, with respect to $\mu^{\prime}$ (see Example 2). By virtue of Theorem 1, the relation

$$
\varepsilon=\mu^{\prime}(X) \leq \mu^{\prime}(Y)=\mu(Y)<\varepsilon
$$

must be valid, which yields a contradiction. This contradiction completes the proof of Theorem 2. 
Remark 1. Clearly, Theorem 2 may be regarded as a generalization of Vitali's theorem [11] stating the existence of subsets of $\mathbb{R}$ nonmeasurable in the Lebesgue sense. Indeed, putting in Theorem $2 E=\mathbb{R}, G=\mathbb{Q}$, and $\mu=$ the Lebesgue measure on $\mathbb{R}$, we directly get Vitali's classical result. Therefore, Theorem 2 may be treated as an abstract version of the above-mentioned Vitali's theorem. Some other versions of Vitali's theorem are presented (see, e.g., [2], [9] and [10]).

In order to show that the notion of the weak metrical transitivity is tightly connected with the existence of absolutely nonmeasurable sets, we need two auxiliary statements.

Lemma 1. Let $E$ be a Polish topological space, and let $W(x, y)(x \in E, y \in E)$ be an equivalence relation on $E$ satisfying the following conditions:

(1) each equivalence class with respect to $W(x, y)$ is a closed subset of $E$;

(2) for any closed set $F \subset E$, the set $W(F)=\{y \in E:(\exists x)(x \in F \&(x, y) \in$ $W)\}$ is Borel in $E$.

If $E / W$ denotes the quotient-set associated with $W$, then there exists a Borel selector of $E / W$.

For the proof of the above-mentioned lemma, see e.g. [1].

Lemma 2. Let $(G, \cdot)$ be a $\sigma$-compact complete metrizable topological group, and let $H$ be a closed subgroup of $G$. Then the family of all right (left) translates of $H$ admits a Borel selector.

Proof. Note first that the given metrizable group $G$ is separable as a union of countably many compact (hence, separable) subspaces. Moreover, applying the classical Baire theorem, we readily infer that $G$ is locally compact. Denote by the symbol $G / H$ the family of all right translates of $H$. Since $H$ is a closed subgroup of $G$, the elements of $G / H$ are also closed in $G$. In view of Lemma 1 , it remains to verify that, for any closed set $F \subset G$, the set

$$
\cup\{H x: x \in G, F \cap H x \neq \emptyset\}=H \cdot F
$$

is Borel in $G$. Indeed, the sets $H$ and $F$ are closed subsets of a $\sigma$-compact space, which implies that they are also $\sigma$-compact. This immediately yields that $H \cdot F$ is $\sigma$-compact, too, and hence is Borel in $G$.

Remark 2. As mentioned above, any $\sigma$-compact complete metrizable topological group $(G, \cdot)$ is a locally compact Polish group (equivalently, is a locally compact group with a countable base). Also, it is well known that if a Polish 
group $(G, \cdot)$ admits a nonzero $\sigma$-finite Borel measure $\mu$ invariant under all left (right) translations of $G$, then $G$ is $\sigma$-compact and locally compact, and $\mu$ coincides with the left (right) Haar measure on $G$.

Now, we are ready to establish the following statement.

Theorem 3. Let $(G, \cdot)$ be an uncountable locally compact Polish group, let $\mu$ denote the left Haar measure on $G$, and let $H$ be a countable subgroup of $G$. We consider $H$ as a group of transformations of $G$. Namely, each $h \in H$ is identified with the mapping $h: G \rightarrow G$ defined by the formula

$$
h(x)=h \cdot x(x \in G) .
$$

The following three assertions are equivalent:

(a) $\mu$ is weakly metrically transitive with respect to $H$;

(b) every $H$-selector in $G$ is absolutely nonmeasurable with respect to $\mu$;

(c) $H$ is non-discrete in $G$.

Proof. The implication (a) $\Rightarrow(\mathrm{b})$ is a straightforward consequence of Theorem 2.

Let us prove the implication (b) $\Rightarrow$ (c). Suppose that a subgroup $H$ of the given group $G$ is discrete. Then it can easily be verified that $H$ is countable and closed in $G$.

Applying Lemma 2, we infer that there exists a Borel $H$-selector in $G$. This selector is $\mu$-measurable, and, therefore, it cannot be absolutely nonmeasurable with respect to $\mu$. We thus conclude that the implication $\neg(\mathrm{c}) \Rightarrow \neg$ (b) is valid. Consequently, we also have (b) $\Rightarrow$ (c).

Finally, suppose that (c) is fulfilled. Let us put $P=\operatorname{cl}(H)$. Clearly, $P$ is an uncountable closed subgroup of $G$. According to Lemma 2, there exists a Borel selector $L$ of the family $G / P$ of all right translates of $P$. Since there are uncountably many pairwise disjoint left translates of $L$, we must have $\mu(L)=0$. Also, it is clear that $P \cdot L=G$. Fix $\varepsilon>0$. Since $\mu(L)=0$, there exists an open set $U \subset G$ such that $L \subset U$ and $\mu(U)<\varepsilon$. Obviously, $P \cdot U=G$. Taking into account that $U$ is open and $H$ is dense in $P$, we deduce that $\cup\{h(U): h \in H\}=H \cdot U=G$, which yields the weak metrical transitivity of $\mu$ with respect to $H$. Thus, (c) $\Rightarrow$ (a) and the proof of the theorem is completed.

Remark 3. It is not difficult to show that the implication (c) $\Rightarrow(b)$ holds for an arbitrary uncountable $\sigma$-compact locally compact topological group $(G, \cdot)$ endowed with the left Haar measure $\mu$. For the sake of completeness, we will 
present a simple proof of this fact, applying the classical argument of Vitali [11] to $G$. Let $H$ be a countable non-discrete subgroup of $G$ (obviously, such subgroups always exist in $G$ ), and let $X$ be an $H$-selector in $G$. Suppose to the contrary that there exists a left $H$-invariant extension $\mu^{\prime}$ of $\mu$ such that $X \in \operatorname{dom}\left(\mu^{\prime}\right)$. Since $\cup\{h X: h \in H\}=G$, we must have $\mu^{\prime}(X)>0$. Further, since $G$ is $\sigma$-compact, there is a compact set $K \subset G$ satisfying the relation

$$
0<\mu^{\prime}(X \cap K) \leq \mu^{\prime}(K)=\mu(K)<+\infty .
$$

Denote $Y=X \cap K$. Let $V$ be a compact neighbourhood of the neutral element of $G$. Since $H$ is non-discrete, the set $H \cap V$ is infinite. Let us represent the last set as an injective sequence $\left\{h_{n}: n \in \mathbf{N}\right\}$. Obviously, the following two relations are satisfied:

(1) $h_{n} Y \cap h_{m} Y=\emptyset$ whenever $n \neq m$;

(2) $\cup\left\{h_{n} Y: n \in \mathbf{N}\right\} \subset V \cdot K$.

Relation (1) directly implies that

$$
\mu^{\prime}\left(\cup\left\{h_{n} Y: n \in \mathbf{N}\right\}\right)=\sum_{n \in \mathbf{N}} \mu^{\prime}\left(h_{n} Y\right)=+\infty .
$$

On the other hand, by virtue of the compactness of $V \cdot K$, relation (2) implies

$$
\mu^{\prime}\left(\cup\left\{h_{n} Y: n \in \mathbf{N}\right\}\right) \leq \mu^{\prime}(V \cdot K)=\mu(V \cdot K)<+\infty .
$$

The obtained contradiction gives us the required result.

In a similar way, Theorem 2 can be applied to the $n$-dimensional Lebesgue measure $\lambda$ on the Euclidean space $\mathbb{R}^{n}$. Indeed, we may consider $\lambda$ as an invariant measure with respect to an arbitrary countable group $H$ of isometric transformations (i.e., motions) of $\mathbb{R}^{n}$. Note that some deep properties of $\lambda$ treated as an $H$-invariant measure on $\mathbb{R}^{n}$ are investigated in paper [6]. In the same paper, various $H$-invariant extensions of $\lambda$ are also studied.

For our purpose, we need several well-known facts from the geometry of Euclidean spaces.

(i) Let $x$ be a point of $\mathbb{R}^{n}$, and let $G$ be a family of motions of $\mathbb{R}^{n}$. If the set $G(x)=\{g(x): g \in G\}$ is relatively compact in $\mathbb{R}^{n}$, then the family $G$ is relatively compact in the topological group of all motions of $\mathbb{R}^{n}$. In particular, if $G$ is a closed subset of the group of all motions of $\mathbb{R}^{n}$, then the set $G(x)$ is closed in $\mathbb{R}^{n}$.

(ii) Let $F$ be a closed subset of $\mathbb{R}^{n}$, and let $G$ be a closed subset of the group of all motions of $\mathbb{R}^{n}$. Then the set $G(F)=\cup\{g(x): g \in G, x \in F\}$ is $\sigma$-compact in $\mathbb{R}^{n}$. Consequently, $G(F)$ is Borel in $\mathbb{R}^{n}$. 
(iii) The group of all motions of $\mathbb{R}^{n}$ acts almost freely in $\mathbb{R}^{n}$ with respect to $\lambda$. Indeed, for any two distinct motions $g$ and $h$ of $\mathbb{R}^{n}$, the set $\left\{x \in \mathbb{R}^{n}\right.$ : $g(x)=h(x)\}$ is contained in some affine hyperplane of $\mathbb{R}^{n}$. Hence, this set is of Lebesgue measure zero.

(iv) Let $G$ be an uncountable group of motions of $\mathbb{R}^{n}$, and let $X$ be a selector of the family of all $G$-orbits. Then either $X$ is nonmeasurable with respect to $\lambda$ or $\lambda(X)=0$.

Note that (iv) readily follows from (iii). More generally, if an uncountable group $G$ of transformations of a set $E$ acts almost freely in $E$ with respect to a $\sigma$-finite $G$-quasi-invariant measure $\mu$ on $E$, then any $G$-selector is either $\mu$-nonmeasurable or has $\mu$-measure zero.

Taking these facts into account, we can formulate for $\lambda$ a direct analog of Theorem 3.

Theorem 4. Let $H$ be a countable group of motions of the Euclidean space $\mathbb{R}^{n}$. The following three assertions are equivalent:

(a) $\lambda$ is weakly metrically transitive with respect to $H$;

(b) every $H$-selector in $\mathbb{R}^{n}$ is absolutely nonmeasurable with respect to $\lambda$;

(c) $H$ is non-discrete in the group of all motions of $\mathbb{R}^{n}$.

The proof of this statement is similar to the proof of Theorem 3 (cf. also [6], Lemmas 2.8 and 2.9).

We are grateful to the referee whose remarks and suggestions were helpful for improving the present paper.

\section{References}

[1] N. Bourbaki, Topologie Générale, Ch. 9, Hermann, Paris, 1960.

[2] J. Cichoń, A. Kharazishvili, B. Weglorz, On sets of Vitali's type, Proc. Amer. Math. Soc., 118(4) (1993), 1221-1228.

[3] P. Gruber, Aspects of convexity and its applications, Expo. Math., 2 (1984), 47-83.

[4] A. B. Kharazishvili, Invariant Extensions of the Lebesgue Measure, Izd. Tbilis. Gos. Univ., Tbilisi, 1983 (Russian).

[5] A. B. Kharazishvili, Topological Aspects of Measure Theory, Izd. Naukova Dumka, Kiev, 1984 (Russian).

[6] A. Krawczyk, P. Zakrzewski, Extensions of measures invariant under countable groups of transformations, Trans. Amer. Math. Soc., 326(1) (1991), 211-226. 
[7] H. Minkowski, Geometrie der Zahlen, Teubner, Leipzig, 1896.

[8] J. C. Oxtoby, Measure and Category, Springer-Verlag, New York, 1971.

[9] S. Solecki, On sets nonmeasurable with respect to invariant measures, Proc. Amer. Math. Soc., 119(1) (1993), 115-124.

[10] S. Solecki, Measurability properties of sets of Vitali's type, Proc. Amer. Math. Soc., 119(3) (1993), 897-902.

[11] G. Vitali, Sul Problema della Misura dei Gruppi di Punti di una Retta, Gamberini e Parmeggiani, Bologna, 1905. 